\title{
Impacto del cobre en la reducción de infecciones intrahospitalarias, mortalidad y gasto en antimicrobianos en una Unidad de Cuidados Intensivo de adultos
}

\author{
Pamela Rivero, Pola Brenner y Patricio Nercelles
}

\begin{abstract}
Hospital Carlos Van Buren, Valparaíso, Chile. Unidad de prevención y control de Infecciones asociadas a la atención en Salud (PR).

Universidad de Valparaíso Valparaíso, Chile. Facultad de Medicina (PB y PN).

Estudio realizado en el Hospital Carlos Van Buren.

Conflictos de interés: No existen. Fuente de financiamiento: Empresa Cobre-Salud.

Recibido: 1 de junio de 2013 Aceptado: 22 de abril de 2014

Correspondencia a: Pamela Rivero Bravo privero3@gmail.com
\end{abstract}

\section{Introducción}

L as infecciones intrahospitalarias (IIH) constituyen una complicación de los cuidados de salud y se ha investigado ampliamente el impacto que éstas tienen tanto en el aumento de la morbilidad y mortalidad como en el costo de los pacientes hospitalizados ${ }^{1}$.

Las IIH están condicionadas por tres factores: el agente etiológico, la vía de transmisión y el paciente susceptible. El personal a cargo de los pacientes constituye un reservorio de microorganismos y puede contribuir a la transmisión de IIH endémicas y epidémicas. Por esta razón, el cumplimiento de la técnica aséptica y las medidas de prevención de IIH en la atención directa son factores clave para el desarrollo o no de las $\mathrm{IIH}^{2}$. El rol del ambiente hospitalario en la propagación de algunas infecciones se encuentra bien establecido, y las evidencias sugieren que superficies hospitalarias contaminadas, en especial las de alto contacto, pueden ser un factor de riesgo para la transmisión de IIH, particularmente en pacientes críticos. En consecuencia, existe creciente reconocimiento que medidas ambientales deben ser un componente esencial de la estrategia global de prevención de $\mathrm{IIH}^{3,4}$.

El cobre es el primer material sólido que recibe la calificación de antimicrobiano por parte de la Agencia de Protección Ambiental (EPA) de Estados Unidos de América (E.U.A.) tras haberse comprobado sus beneficios en la reducción constante de la contaminación microbiana en superficies. Pruebas de laboratorio han comprobado las propiedades naturales del cobre y sus aleaciones, mostrando que reduce significativamente la contaminación con microorganismos tales como Staphylococus aureus, en comparación con acero inoxidable que constituye la superficie más utilizada en los hospitales ${ }^{5-9}$.

En Chile, el Hospital Salvador Allende de Calama, formó parte de un estudio multicéntrico, cuyo objetivo fue determinar la disminución de carga bacteriana en unidades de cuidados intensivos, mediante la cobrización de los elementos de la unidad del paciente. La investigación fue realizada durante 30 semanas y se cobrizaron las barandas de la cama del paciente, las mesas, el lápiz del monitor y la silla de visitas. Se observó una reducción del recuento bacteriano en todos los elementos con cobre, menor recuento bacteriano total en las habitaciones y ausencia de Staphylococcus aureus resistente a meticilina y Enterococcus spp resistente a vancomicina en las salas cobrizadas $^{10,11}$.

Recientemente se llevó a cabo un estudio que demostró el impacto del cobre en la reducción de infecciones. Este fue realizado durante el año 2010 y 2011 en tres hospitales de E.U.A. Se compararon las tasas de infección en los pacientes que ingresaron en salas con cobre y en aquellas sin cobre, observándose una disminución de $40 \%$ en las tasas de IIH (8,95 por mil días paciente en unidades de 
cobre versus 15,16 por mil días paciente en unidades sin cobre, OR: 0,5 (95\% IC O,41-0,73), $\mathrm{p}=0,00003^{12}$.

El objetivo del presente trabajo es determinar la efectividad del cobre en la disminución de infecciones intrahospitalarias, mortalidad asociada y los gastos de antimicrobianos asociados a éstas en una Unidad de Cuidados Intensivos de un hospital de alta complejidad.

\section{Material y Método}

Ensayo clínico, donde los pacientes ingresaron al azar a las camas la Unidad de Cuidados Intensivos de Adultos del Hospital Carlos Van Buren, según disponibilidad de ellas, en el período mayo de 2011 a mayo de 2012. Previo al inicio del estudio, se cobrizaron la mitad de las unidades de pacientes (7 de 14 unidades) revistiendo cada unidad con cobre aleación UNS 11000 (99\% cobre) que equivale a aproximadamente $80 \%$ de las áreas más tocadas por los pacientes (cuatro barandas de la cama, mesa del paciente y dos portasueros). Sólo no se cobrizó el velador y lápiz del monitor. En ambos grupos se estudió, prospectivamente, la aparición de IIH asociada a procedimientos invasores (neumonía asociada a ventilación mecánica, infección urinaria asociada a catéter urinario y bacteriemia asociada a catéter venoso central), mortalidad asociada a estas infecciones y el gasto en antimicrobianos durante su estadía. Además se consignaron datos demográficos y antecedentes de importancia de los pacientes, tales como edad, sexo y antecedentes mórbidos. No fue posible consignar el estado de gravedad (APACHE u otro) al ingreso a UCI ya que no se realiza esta clasificación en forma rutinaria. La limpieza y desinfección de las superficies cobrizadas se realizó con solución de ácido cítrico $0,6 \%$ (recomendación del fabricante) y en las no cobrizadas con amonio cuaternario. Durante el período que duró el estudio no se establecieron cambios en las medidas generales de prevención de IIH en la UCI.

La indicación de antimicrobianos fue responsabilidad de cada médico; la institución no cuenta con protocolos escritos de tratamientos según tipo de infección, pero sí las indicaciones de antimicrobianos de última generación o restringidos, son visadas por infectólogos.

A fin de establecer el número de pacientes a ser incluidos, se estimó una muestra de pacientes en base a las tasas obtenidas del sistema de vigilancia de IIH del hospital. Se determinó un poder de $80 \%$, un intervalo de confianza de $95 \%$ y una capacidad de detectar un riesgo relativo de 2 , lo que determinó un tamaño de muestra de 1.734 pacientes, de los cuales 867 pacientes se encontrarían expuestos a la intervención y 867 sin la intervención. Considerando que ingresan anualmente 497 pacientes a la UCI (según información del hospital al año 2010), se estimó necesario un período de seguimiento de 24 meses para completar la muestra.
Para el análisis de los datos, se compararon las tasas de IIH en los grupos con y sin cobre, utilizando el programa Stata 11@ para el análisis de las diferentes variables mediante las pruebas T de Student, $\chi^{2}$, Mann Whitney. Para el análisis de sobrevida libre de infección se utilizó la prueba de Kaplan-Meier y test de Log-Rank. Para el análisis de las tasas de incidencia se utilizó la regresión lineal de Poisson. Se estableció el nivel de significancia en un valor de $\mathrm{p}<0,05$.

Los criterios de inclusión fueron la permanencia de más de $24 \mathrm{~h}$ en la Unidad de Cuidados intensivos. Antes de la realización del estudio se obtuvo la aprobación del Comité de Ética del hospital.

\section{Resultados}

Durante el período de trece meses del estudio ingresaron 538 pacientes, de los cuales 97 no cumplían con el criterio de inclusión de permanecer más de $24 \mathrm{~h}$ en la Unidad de Cuidados Intensivos. Hubo pérdida de seguimiento de un paciente por extravío de ficha clínica.

En total ingresaron 440 pacientes al estudio, 217 pacientes $(49,3 \%)$ en el grupo sin cobre y 223 en el grupo con cobre $(50,7 \%)$. Los grupos fueron similares en edad, antecedentes mórbidos, sexo, número de diagnósticos $\mathrm{y}$ fallecidos (Tabla 1).

Se notificó un total de $73 \mathrm{IIH}$, de las cuales $50,7 \%$ correspondieron a pacientes en unidades sin cobre y $49,3 \%$ a pacientes internados en unidades con cobre. El 42,5\% de las IIH correspondió a neumonía asociada a ventilación mecánica, el 39,7\% a infección urinaria asociada a catéter urinario permanente, y el 17,8\% a bacteriemia asociada a catéter venoso central (Tabla 2).

En el grupo con cobre hubo 189 pacientes en ventilación mecánica, (2.083 días de exposición y promedio de 9,3 días por paciente), 221 pacientes con catéter urinario a permanencia (2.350 días de exposición y promedio 10,6 días por paciente) y 214 pacientes con catéter venoso central (2.226 días de exposición y promedio 10,4 días por paciente).

En el grupo sin cobre hubo 177 pacientes con ventilación mecánica, (1.977 días de exposición y promedio 10,8 días por paciente), 216 pacientes con catéter urinario permanente (2.216 días de exposición y promedio 10,2 días por paciente) y 210 pacientes con catéter venoso central (2.045 días de exposición y promedio de 9,6 días por paciente).

Las tasas de neumonía asociada a ventilación mecánica e infección urinaria asociada a catéter urinario fueron similares en ambos grupos, sin diferencias estadísticamente significativas entre ellos. La tasa de bacteriemia asociada a catéter venoso central fue 1,7 veces mayor en el grupo sin cobre $(3,9$ por mil días de exposición en el grupo sin 
cobre y 2,2 por mil días de exposición en el grupo con cobre), diferencia no significativa (Tabla 3 ).

Se evaluó el efecto del cobre en la aparición de IIH. En el grupo internado en unidades con cobre, el día promedio de aparición de neumonía fue el día 12 de hospitalización, en infección urinaria el día 22 y en bacteriemia el día 9. En el grupo internado en unidades sin cobre se evidenciaron los mismos días para neumonía y bacteriemia, pero se observó una aparición más temprana en infección urinaria

Tabla 1. Características de la población en estudio, UCI adultos. Hospital Van Buren, Valparaíso. 2011-2012

\begin{tabular}{|c|c|c|c|c|c|}
\hline Variables & \multicolumn{2}{|c|}{$\begin{array}{l}\text { Unidad con cobre } \\
\text { (n: 223) }\end{array}$} & \multicolumn{2}{|c|}{$\begin{array}{l}\text { Unidad sin cobre } \\
\text { (n: 217) }\end{array}$} & Valor $p$ \\
\hline \multicolumn{6}{|l|}{ Sexo } \\
\hline Femenino & 109 & $(48,9 \%)$ & 97 & $(44,7 \%)$ & \multirow{2}{*}{0,3} \\
\hline Masculino & 114 & $(51,1 \%)$ & 120 & $(55,3 \%)$ & \\
\hline Edad promedio & 51 & $(15-91)$ & 51,1 & $(15-86)$ & 0,7 \\
\hline \multicolumn{6}{|l|}{ Antecedentes mórbidos } \\
\hline Diabetes mellitus & & 33 & & 27 & 0,4 \\
\hline Hipertensión arterial & & 86 & & 78 & 0,5 \\
\hline Tabaquismo & & 29 & & 22 & 0,3 \\
\hline Alcoholismo & & 14 & & 10 & 0,4 \\
\hline Otros & & 20 & & 23 & 0,5 \\
\hline Promedio de diagnósticos al ingreso & & 2 & & 2 & 0,8 \\
\hline
\end{tabular}

Tabla 2. Frecuencia de infecciones en UCI adultos según localización. Hospital Van Buren, Valparaíso. 2011-2012

\begin{tabular}{lcccc} 
Localizaciones & $\begin{array}{c}\text { Unidad } \\
\text { con cobre }\end{array}$ & $\begin{array}{c}\text { Unidad } \\
\text { sin cobre }\end{array}$ & Total & $\%$ \\
Neumonía asociada a ventilación mecánica & 16 & 15 & 31 & 42,5 \\
Infección urinaria asociada a catéter urinario & 15 & 14 & 29 & 39,7 \\
Bacteriemia asociada a catéter venoso central & 5 & 8 & 13 & 17,8 \\
Total & 36 & 37 & 73 & 100 \\
\hline
\end{tabular}

(tiempo promedio de 18 días). Esta diferencia no fue estadísticamente significativa $(\mathrm{p}=0,9)$ (Tabla 4).

Se evaluó la mortalidad asociada a IIH en 96 de los 97 pacientes fallecidos por neumonía asociada a ventilación mecánica y bacteriemia asociada a catéter venoso central en el período (una de las fichas no pudo ser encontrada en archivo por causas externas a la investigación). De los 96 pacientes analizados, en dos casos la infección fue la causa de la muerte. En nueve, la infección contribuyó a la muerte y en el resto la muerte no estuvo asociada a la infección. No hubo diferencias significativas entre grupo con cobre y sin cobre (p: 0,9) (Tabla 5).

\section{Gasto en antimicrobianos}

Se registró el gasto total en antimicrobianos de los pacientes en los grupos con y sin cobre. Se calculó el gasto de antimicrobianos en pacientes con IIH en ambos grupos. Se separaron por tipo de antimicrobiano y el número de dosis administradas (Tabla 6).

Se observó un leve mayor gasto total de antimicrobianos en los pacientes ubicados en las unidades sin cobre versus aquellos en unidades con cobre y la mayor diferencia se encontró en el uso de antimicrobianos de pacientes con IIH en ambos grupos (Tabla 7). No se encontraron diferencias en el tipo de agentes etiológicos en ambos grupos (Tabla 8).

\section{Discusión}

La contaminación de las superficies de alto contacto, se han asociado a la transmisión de IIH en particular producidas por agentes multiresistentes y Clostridium difficile, debido a su prolongada sobrevida en el ambiente inanimado y la posibilidad de colonización de las manos del personal que pueden diseminar estos microorganismos en el ambiente o colonizar otros pacientes. Por otra parte, la limpieza manual de estas superficies ha demostrado deficiencias en términos de reducción de la contaminación microbiana de estas superficies y dificultad en estandarizarla, observándose por ende gran variabilidad en los distintos establecimientos ${ }^{3,4}$.

Tabla 3. Tasas de infección intrahospitalaria en pacientes internados en unidades con cobre y sin cobre. Hospital Van Buren, Valparaíso. 2011-2012

Localizaciones

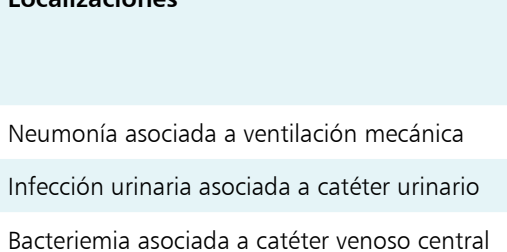

Unidad con cobre

n de IIH
Días de Tasa por mil días exposición de exposición

\begin{tabular}{lll}
16 & 2.083 & 7,7 \\
15 & 2.350 & 6,4 \\
5 & 2.226 & 2,2 \\
\hline
\end{tabular}

\section{Unidad sin cobre}

Días de
exposición

7
6
6
3


Tabla 4. Día promedio de aparición de la primera infección intrahospitalaria Hospital Van Buren, Valparaíso. 2011-2012

\begin{tabular}{|lccc|} 
& $\begin{array}{c}\text { Unidad } \\
\text { con cobre }\end{array}$ & $\begin{array}{c}\text { Unidad } \\
\text { sin cobre }\end{array}$ & Valor $\mathbf{p}$ \\
$\begin{array}{l}\text { Neumonía asociada a ventilación } \\
\text { mecánica }\end{array}$ & 12 & 12 & ND* \\
$\begin{array}{l}\text { Infección urinaria asociada a catéter } \\
\text { urinario }\end{array}$ & 22 & 18,3 & 0,9 \\
$\begin{array}{l}\text { Bacteriemia asociada a catéter } \\
\text { venoso central }\end{array}$ & 9,6 & 9,4 & ND* $^{*}$ \\
\hline *Valor no calculado. & & & \\
\hline
\end{tabular}

Tabla 6. Tipo de antimicrobianos utilizados en unidades con y sin cobre. Hospital Van Buren, Valparaíso. 2011-2012

\begin{tabular}{|c|c|c|c|c|}
\hline & Unida & d con cobre & Unida & d sin cobre \\
\hline & $\begin{array}{c}n \\
\text { dosis }\end{array}$ & $\begin{array}{c}\text { Gasto } \\
\text { total (US\$) }\end{array}$ & $\begin{array}{c}n \\
\text { dosis }\end{array}$ & $\begin{array}{c}\text { Gasto } \\
\text { total(US\$) }\end{array}$ \\
\hline Amikacina 100 mg & 23 & 11,8 & 61 & 31,2 \\
\hline Amikacina 500 mg & 56 & 39,6 & 109 & 77,0 \\
\hline $\begin{array}{l}\text { Amoxicilina/sulbactam } \\
1.000 / 500 \mathrm{mg}\end{array}$ & 3 & 30,2 & 100 & $1.007,3$ \\
\hline Ampicilina $500 \mathrm{mg}$ & 40 & 4,9 & 472 & 57,9 \\
\hline Cefazolina $1 \mathrm{~g}$ & 155 & 86,9 & 20 & 11,2 \\
\hline Cefepime $1 \mathrm{~g}$ & 220 & $1.582,9$ & 151 & $1.086,4$ \\
\hline Cefotaxima $1 \mathrm{~g}$ & 1.157 & 932,8 & 901 & 726,4 \\
\hline Ceftazidima $1 \mathrm{~g}$ & 37 & 79,2 & 33 & 70,7 \\
\hline Ceftriaxona $1 \mathrm{~g}$ & 590 & 410,8 & 731 & 508,9 \\
\hline Clindamicina 300 mg & 756 & $2.962,8$ & 752 & 2947 \\
\hline Clindamicina 600 mg & 222 & 175,7 & 213 & 168,6 \\
\hline Cloxacilina 500 mg & 885 & 470,2 & 1.054 & 559,8 \\
\hline Cotrimoxazol & 363 & 938,7 & 277 & 716,3 \\
\hline Ertapenem $1 \mathrm{~g}$ & 47 & $4.691,3$ & 26 & $2.595,2$ \\
\hline Gentamicina 80 mg & 40 & 3,2 & 51 & 4,1 \\
\hline $\begin{array}{l}\text { Imipenem/cilastatina } \\
500 \mathrm{mg}\end{array}$ & 1.054 & 14.598 & 794 & 10.997 \\
\hline Meropenem $500 \mathrm{mg}$ & 397 & $4.674,3$ & 460 & 5416 \\
\hline Meropenem $1 \mathrm{~g}$ & 485 & $21.679,1$ & 714 & 3.1915 \\
\hline Metronidazol $250 \mathrm{mg}$ & 564 & 11,9 & 724 & 15,3 \\
\hline Metronidazol $500 \mathrm{mg}$ & 581 & 463,5 & 635 & 506,6 \\
\hline Moxifloxacina $400 \mathrm{mg}$ & 69 & 2.867 & 95 & $3.947,3$ \\
\hline Penicilina G 2.000.000 UI & 152 & 28,7 & 0 & 0 \\
\hline Sulbactam/ampicilina $1,5 \mathrm{~g}$ & 78 & 1.167 & 352 & 5267 \\
\hline Sulbactam/cefoperazona $1,5 \mathrm{~g}$ & 270 & $1.560,4$ & 650 & $3.756,5$ \\
\hline Vancomicina $1 \mathrm{~g}$ & 995 & $6.013,5$ & 830 & $5.016,3$ \\
\hline Total & & $65.484,4$ & & 77.405 \\
\hline
\end{tabular}

Tabla 5. Mortalidad asociada a infección intrahospitalaria. Hospital Van Buren, Valparaíso. 2011-2012

\begin{tabular}{|lccc|} 
& $\begin{array}{c}\text { Unidad } \\
\text { con cobre } \\
\text { (n: 51) }\end{array}$ & $\begin{array}{c}\text { Unidad } \\
\text { sin cobre } \\
\text { (n: 45) }\end{array}$ & Valor \\
La infección fue la causa de la muerte & 2 & 0 & \\
La infección contribuyó a la muerte & 5 & 4 & 0,9 \\
La infección no estuvo relacionada con la muerte & 44 & 41 & \\
\hline
\end{tabular}

Tabla 7. Gasto de antimicrobianos utilizados en pacientes en unidades con y sin cobre, según presencia de infección intrahospitalaria. Hospital Van Buren, Valparaíso. 2011-2012

\begin{tabular}{|c|c|c|c|c|c|c|}
\hline \multirow{2}{*}{$\begin{array}{l}\text { Tipo de } \\
\text { paciente }\end{array}$} & \multicolumn{2}{|c|}{ Unidad con cobre } & \multicolumn{2}{|c|}{ Unidad sin cobre } & \multicolumn{2}{|c|}{ Total } \\
\hline & $\begin{array}{l}\text { Gasto } \\
\text { US\$ }\end{array}$ & $\begin{array}{l}\text { Promedio } \\
\text { paciente }\end{array}$ & $\begin{array}{l}\text { Gasto } \\
\text { US\$ }\end{array}$ & $\begin{array}{l}\text { Promedio } \\
\text { paciente }\end{array}$ & $\begin{array}{l}\text { Gasto } \\
\text { US\$ }\end{array}$ & $\begin{array}{c}\text { Promedio } \\
\text { paciente }\end{array}$ \\
\hline $\begin{array}{l}\text { Paciente } \\
\text { con infección } \\
\text { intrahospitalaria }\end{array}$ & 25.694 & 713,7 & 38.062 & $1.057,3$ & 63.756 & 873,4 \\
\hline $\begin{array}{l}\text { Pacientes } \\
\text { sin infección } \\
\text { intrahospitalaria }\end{array}$ & 39.791 & $1.105,3$ & 39.343 & $1.092,9$ & 79.134 & $1.084,0$ \\
\hline Total & 65.485 & $1.819,0$ & 77.405 & $2.150,1$ & 142.890 & 1957,4 \\
\hline
\end{tabular}

Tabla 8. Agentes etiológicos de infecciones en unidades con y sin cobre. Hospital Van Buren, Valparaíso. 2011-2012

\begin{tabular}{|lccc|}
\hline Ulebsiella pneumoniae & $\begin{array}{c}\text { Unidad con } \\
\text { cobre }\end{array}$ & $\begin{array}{c}\text { Unidad sin } \\
\text { cobre }\end{array}$ & Total \\
Escherichia coli & 8 & 12 & 20 \\
Staphylococcus aureus & 5 & 7 & 12 \\
Acinetobacter baumannii & 5 & 7 & 12 \\
Enterococcus faecalis & 5 & 5 & 10 \\
Pseudomonas aeruginosa & 2 & 3 & 5 \\
Haemophilus influenzae & 3 & 1 & 4 \\
Otros & 2 & 1 & 3 \\
Total & 6 & 1 & 7 \\
\hline
\end{tabular}

El efecto antimicrobiano del cobre en superficies de alto contacto ha sido demostrado en publicaciones que reportan leve disminución y hasta $83 \%$ de la carga microbiana comparada con superficies no cobrizadas $^{5-16}$. Por lo anterior, esta tecnología puede significar un aporte en la prevención y control de IIH en el paciente crítico, dado que actúa independiente de conductas o motivación del personal del equipo de salud. Las superficies cobrizadas constituyen una forma pasiva de reducir la carga microbiana que no requiere mayores esfuerzos de implementación. 
Excepto la publicación de Salgado y cols ${ }^{17}$, no se ha podido demostrar el impacto en la reducción de los riesgos de infecciones. En nuestro estudio, no pudimos demostrar diferencias significativas en las tasas de IIH estudiadas a pesar que la tasa de bacteriemia asociada a catéteres venosos centrales fue 1,7 veces más alta en pacientes hospitalizados en unidades no cobrizadas. La principal limitación de esta investigación fue no alcanzar el tamaño de muestra calculado, por lo que la ausencia de diferencias puede atribuirse a este hecho. Además, no se incluyeron infecciones en localizaciones no asociadas a procedimientos invasores, tales como infecciones gastrointestinales, que se han asociado en varios estudios a contaminación ambiental $^{12}$. Sin embargo, hubo una diferencia en el gasto de antimicrobianos y mucho mayor utilización de antimicrobianos de amplio espectro que se utilizan en general en tratamientos de infecciones producidas por agentes multiresistentes, en pacientes hospitalizados en unidades no cobrizadas.

Aunque hubo algunas diferencias en el número y tipo de bacterias en los pacientes de áreas cobrizadas y no cobrizadas, éstas no corresponden a grupos bacterianos que explicaran diferencias en la supervivencia de las superficies de las unidades.

Otra de las limitaciones es que la asignación a las unidades cobrizadas no fue ciega debido a que la intervención se distingue a simple vista. Lo anterior, puede haber significado prácticas distintas en los médicos que pudieran haber influido en los resultados.

Finalmente algunas de las superficies cobrizadas se deterioraron durante el estudio lo que pudo haber provocado que pacientes considerados en el grupo con cobre, no hayan estado totalmente circunscritos a una unidad cobrizada.

A pesar de las limitaciones, pudimos observar diferencias no significativas en la tasa de bacteriemias asociadas a catéteres venosos centrales y diferencias en el gasto de antimicrobianos en los pacientes hospitalizados en unidades cobrizadas. Este es el primer estudio de estas características que se realiza en Latinoamérica y sus resultados podrán servir de base para estudios futuros.

Dado que Chile es un país cuprífero, ciertamente esta investigación abre una oportunidad en el uso del cobre para reducir contaminación ambiental que puede tener impacto en la disminución de infecciones en pacientes de UCI.
Agradecimientos. A Gabriel Cavada por su invaluable ayuda en el análisis estadístico. Al Hospital Carlos Van Buren por su apoyo y en especial al equipo de la Unidad de Cuidado Intensivo adulto por su colaboración en esta investigación.

\section{Resumen}

Introducción: Las infecciones nosocomiales incrementan la mortalidad y costos en las instituciones de salud. El revestimiento con cobre, de superficies de alto contacto en la unidad clínica en torno a los pacientes, reduce la colonización bacteriana de las mismas. Objetivo: Determinar el impacto del revestimiento de las superficies con cobre en la disminución de las infecciones intrahospitalarias, la mortalidad asociada a las infecciones intrahospitalarias y los costos en antimicrobianos en pacientes hospitalizados en UCI adultos en el Hospital Carlos Van Buren. Pacientes y Métodos: Estudio prospectivo, comparativo, mayo de 2011-mayo de 2012. Asignación aleatoria de pacientes adultos ingresados en UCI, que permanecieran al menos por $24 \mathrm{~h}$ en dicha unidad, a unidades de aislamiento recubiertas (n: 7) o no recubiertas con cobre (n: 7). Resultados: Ingresaron al estudio 440 pacientes, 217 pacientes $(49,3 \%)$ en el grupo sin cobre y 223 en el grupo con cobre $(50,7 \%)$. No se encontraron diferencias en la frecuencia de infecciones intrahospitalarias en ambos grupos. Tampoco se encontraron diferencias significativas en las tasas de neumonía asociada a ventilación mecánica $(\mathrm{p}=0,9)$, infección urinaria asociada a catéter urinario $(\mathrm{p}$ $=0,9)$ y bacteremias asociada a catéter venoso central $(\mathrm{p}=$ $0,3)$. Tampoco se encontraron diferencias en la sobrevida libre de infección $(\mathrm{p}=0,9)$. Se encontró un gasto menor de antimicrobianos en pacientes atendidos en unidades revestidas con cobre. Durante el período del estudio no se completó el tamaño de muestra y las diferencias no significativas podrían deberse a este hecho. Conclusión: El uso del cobre como revestimiento de las superficies hospitalarias en UCI, mostró diferencia en la tasa de bacteriemia asociada a dispositivos venosos, aunque no significativa, y no mostró diferencia en neumonías e infecciones urinarias. Las diferencias no significativas pueden deberse a que no se completó el tamaño de la muestra. Se observó un mayor gasto de antimicrobianos en pacientes de unidades no cobrizadas, lo que plantea una nueva área de investigación.

\section{Referencias bibliográficas}

1.- Brenner P, Nercelles P, Pohlenz M. Costo de las infecciones intrahospitalarias en hospitales chilenos de alta y mediana complejidad. Rev Chilena Infectol 2003; 20: 285-90.

2.- Pérez L, Zurita I, Pérez N, Patiño N,
Calvimonte O. Infecciones intrahospitalarias: agentes, manejo actual y prevención. Rev Cient Cienc Med 2010; 13: 90-4.

3.- Bhalla A, Pultz N J, Gries D M, Ray A J, Eckstein E C, Aron D C, et al. Acquisition of nosocomial pathogens on hands after contact with environmental surfaces near hospitalized patients. Infect Control Hosp Epidemiol 2004; 25: 164-7.

4.- Blythe D, Keenlyside D, Dawson S J, Galloway A. Environmental contamination due to methicillin-resistant Staphylococcus aureus (MRSA). J Hosp Infect 1998; 38: 67-9.

5.- Lewis A, Keevil C W. The viability of 
antimicrobial copper as a hygienic material for HVAC System components. Cooper Development Association Inc and ICA, Ltda. 2004.

6.- ECOSEA Innovation in Aquaculture (ECOSEA). 2012. Disminución de tasas de infección en hospitales e institutos de salud. En ECOSEA. [En línea]. 2012. Disponible en http://www.ecosea.cl/pdfs/libro_bactericida.pdf. [accedido 19 de septiembre de 2012].

7.- Rai S, Hirsch B E, Attaway H H, Nadan R, Fairey S, Hardy J, et al. Evaluation of the antimicrobial properties of copper surfaces in an outpatient infectious disease practice. Infect Control Hosp Epidemiol 2012; 33 (2): 200-1.

8.- Schmidt M G, Attaway Iii H H, Fairey S E, Steed L L, Michels H T, Salgado C D. Copper continuously limits the concentration of bacteria resident on bed rails within the intensive care unit. Infect Control Hosp Epidemiol 2013; 34 (5): 530-3.

9.- Karpanen T J, Casey A L, Lambert P A, Cookson B D, Nightingale P, Miruszenko L, et al. The antimicrobial efficacy of copper alloy furnishing in the clinical environment: a crossover study. Infect Control Hosp Epidemiol 2012; 33 (1): 3-9.

10.- Prado V, Durán C, Crestto M. Effectiveness of copper contact surfaces in reducing the microbiol burden in the intensive care unit of Hospital del Cobre, Calama, Chile. Presented at the $14^{\text {th }}$ International Conference on Infectious Diseases, Poster 56.044, Miami, 11 March 2011.

11.- Noyce J O, Michels H, Keevil C W. Potential use of copper surfaces to reduce survival of epidemic methicillin-resistant Staphylococcus aureus in the healthcare environment. J Hosp Infect 2006; 63: 289-97.

12.- Weaver L, Michels HT, Keevil CW. Survival of Clostridium difficile on copper and steel: future options for hospital higiene. J Hosp Inf 2008; 68: $145-51$.

13.- Schmidt M G, Attaway H H, Fairey S E, Steed L L, Michels H T, Salgado C D. Copper continuously limits the concentration of bacteria resident on bed rails within the intensive care unit. Infect Control Hosp Epidemiol 2013; 34 (5): 530-3.

14.- Schmidt M G, Attaway H H, Sharpe P A, John J Jr, Sepkowitz K A, Morgan A, et al. Sustained reduction of microbial burden on common hospital surfaces through introduction of copper. J Clin Microbiol 2012; 50 (7): 2217-23.

15.- Karpanen T J, Casey A L, Lambert P A, Cookson B D, Nightingale P, Miruszenko L, et al. The antimicrobial efficacy of copper alloy furnishing in the clinical environment: a crossover study. Infect Control Hosp Epidemiol 2012; 33 (1): 3-9.

16.- Prado V, Vidal R, Durán C. Aplicación de la capacidad bactericida del cobre en la práctica médica. Rev Med Chile 2012; 140: 1325-32.

17.- Salgado C D, Sepkowitz K A, John J F, Cantey J R, Attaway H H, Freeman K D, et al. Copper surfaces reduce the rate of healthcareacquired infections in the intensive care unit. Infect Control Hosp Epidemiol 2013; 34 (5): 479-86. 